\title{
Electric field-enhanced assembly of polyelectrolyte composite membranes
}

\author{
Guojun Zhang ${ }^{\mathrm{a}, *}$, Xue Gao ${ }^{\mathrm{a}}$, Shulan $\mathrm{Ji}^{\mathrm{a}}{ }^{\text {, Zhongzhou Liu }}{ }^{\mathrm{b}}$ \\ ${ }^{a}$ Center for Membrane Technology, College of Environmental and Energy Engineering, \\ Beijing University of Technology, Beijing 100022, PR China \\ ${ }^{\mathrm{b}}$ Research Center for Eco-Environmental Sciences, Chinese Academy of Sciences, \\ Beijing 100085, PR China
}

Received 21 August 2007; received in revised form 8 September 2007; accepted 14 September 2007 Available online 21 September 2007

\begin{abstract}
In this paper, a novel method was developed to enhance the assembly of polyelectrolyte composite membranes by inducing an electric field during electrostatic adsorption process. The hydrolyzed polyacrylonitrile (PAN) ultrafiltration (UF) membrane was placed in between a capacitor setup. The polyethyleneimine (PEI) was compulsorily assembled on the PAN support under the action of external electric force. Subsequently, the polyelectrolyte composite membranes were evaluated by pervaporation separation of water and alcohol mixture. The membrane obtained with only one PEI layer had a separation factor of 304 and a permeate flux of $512 \mathrm{~g} / \mathrm{m}^{2} \mathrm{~h}\left(75^{\circ} \mathrm{C}\right)$ for pervaporation of $95 \mathrm{wt} \%$ ethanol-water mixture. An atomic force microscopy was also used to observe the microtopographical changes. The regularity of the membranes assembled by the new method was also improved in comparison with the membrane assembled by a dynamic layer-by-layer adsorption.
\end{abstract}

(c) 2007 Elsevier B.V. All rights reserved.

Keywords: Polyelectrolyte composite membrane; Electric field; Dynamic layer-by-layer process; Pervaporation; Water/alcohol mixture

\section{Introduction}

Electrostatic layer-by-layer (LBL) assembly is a demonstrated technique for making nanostructured polymer composites from polyelectrolytes and has attracted much interest in recent years [1-3]. This method is based on the alternating electrostatic layer-by-layer adsorption of oppositely charged polyelectrolytes. The membranes obtained by LBL adsorption are especially suitable for the pervaporation separation of various alcohol/water mixtures. However, LBL assembly is a time-consuming process since a large number of depositions of anionic/cationic polyelectrolytes are usually needed in order to achieve a high selectivity. For example, as many as 60 bilayers had to be deposited to obtain membranes with sufficient properties in Tieke and co-workers' studies [1-3]. Therefore, a few attempts have been made to develop new enhanced LBL technique to reduce the number of deposition cycles required. For instances, Sullivan and Bruening [4] combined the versatility of

\footnotetext{
* Corresponding author. Tel.: +86106739 2393; fax: +861067391983.

E-mail address: zhanggj@bjut.edu.cn (G. Zhang).
}

LBL adsorption with covalent interlayer cross-linking to assemble ultrathin polyimide pervaporation membranes. A modified single-sided deposition technique has been developed by Zhu et al. [5] and a good permselectivity was achieved with less than 10 cycles of deposition. More recently, we have reported [6-7] on the dynamic LBL adsorption technique, which would allow reduction of the deposition cycles. This depends on the nondefective and selective layers formed within a few cycles under a certain pressure. In addition to the deposition cycles, the regularity of layer-by-layer structural order might play a significant role in improving the pervaporation capability of membranes obtained. This study therefore seeked to develop a method to regulate the structure order by using a manipulative external force.

Since our previous studies [6-7] have demonstrated that the reduction in the cycles of polyelectrolyte deposition can be achieved while still retaining a very good selectivity by combining the layer-by-layer electrostatic force with a certain external force, it was thought that the assembly could possibly be enhanced under the action of electric field as the polyelectrolytes have their own charges. A schematic diagram, which simply describes the principles of electric field-enhanced 


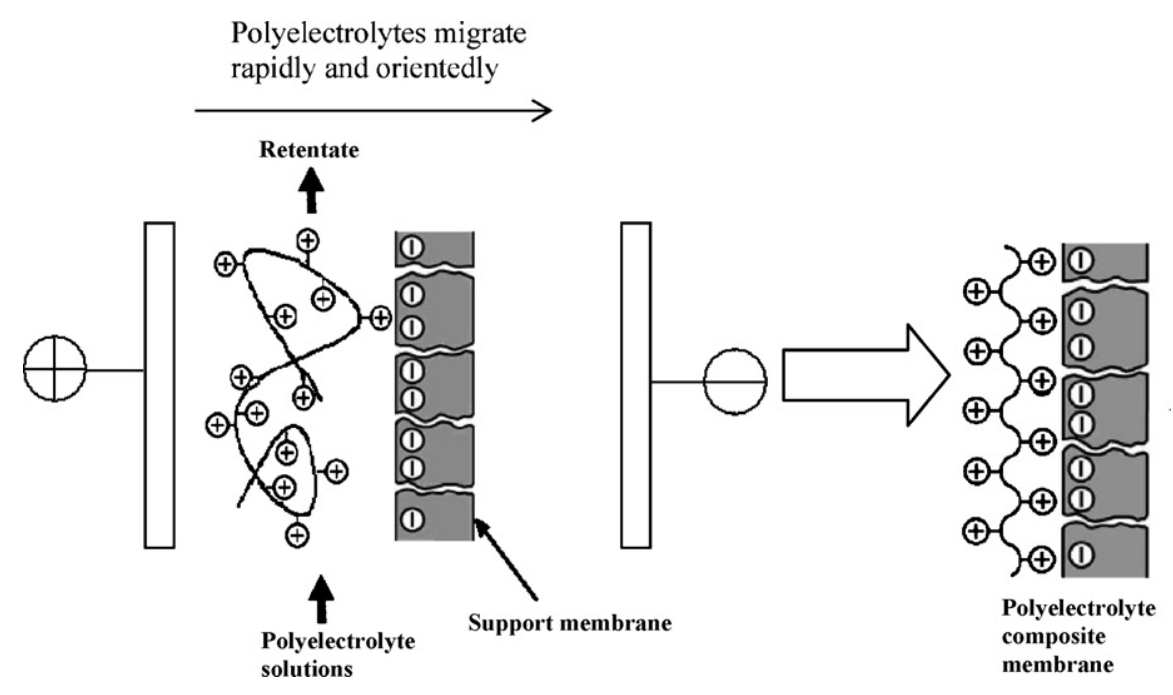

Fig. 1. Principles of electric field-enhanced assembly of polyelectrolyte composite membranes.

assembly of polyelectrolyte composite membranes, is shown in Fig. 1. As shown in Fig. 1, two electrodes are positioned, one on either side parallel to the membrane. Applying a voltage to the electrodes generates an electric field. The field vector perpendicular to the membrane provokes a migration of charged polyelectrolytes towards the electrode with the opposite sign and in turn to obtain the uniform and dense membrane more rapidly. Meanwhile, it was also expected that the layer-by-layer structure order could be regulated due to the oriented characteristics of the polyelectrolytes under DC electric field.

In this study, a crossflow system was proposed to enhance the assembly of polyelectrolyte membranes by using a capacitor to generate DC electric field. The hydrolyzed polyacrylonitrile (PAN) ultrafiltration (UF) membrane was used as the support and placed in between the capacitor setup. The polyethyleneimine (PEI), used as a model polycation, was compulsorily assembled on the PAN UF membrane under DC electric field. The pervaporation separation of $95 \mathrm{wt} \%$ ethanol-water mixture was conducted to compare the effects of extern field forces on membrane performances. Additionally, microtopographical changes of the membrane surfaces were compared by using an atomic force microscopy.

\section{Experimental}

A schematic diagram of experimental setup is shown in Fig. 2(a). It is comprised of a circulation system, a DC power supply unit and a crossflow capacitor cell, in which the flat sheet membrane is loaded. The polyelectrolyte solution is pumped from the recycle tank to the capacitor cells. The retentate is returned to the feed tank whereas permeate is collected into a beaker. For comparison purposes, a dead-end filtration cell as our previous studies mentioned [6] was also used to conduct the dynamic PEI deposition. In addition, the crossflow dynamic assembly without electric field was also conducted as a control experiment.

This capacitor membrane cell constructed of polyvinyl chloride (PVC) is presented in Fig. 2(b). The assembly of the cell consists of two pieces (A and B) into which the electrodes were inserted. The anode deployed is a stainless steel sheet mounted in a parallel position just near the flat sheet membrane in the concentrate compartment. The cathode, mounted in the permeate compartment, is a porous, stainless steel disk inserted into the PVC body cell. Both electrodes are packed with insulating tapes before use. The sizes of the capacitor and the effective membrane are $280 \mathrm{~mm} \times 140 \mathrm{~mm}$ and $220 \mathrm{~mm} \times 80 \mathrm{~mm}$, respectively. The distance between cathode and anode is $22 \mathrm{~mm}$, while the distance between the anode and substrate membrane is $12 \mathrm{~mm}$. The two electrodes of each cell are connected to a DC power supply.

The assembly of polyelectrolyte membranes was performed with a flat-sheet PAN UF membrane, which was provided by the Research Center for Eco-Environmental Sciences, Chinese Academy of Sciences. The molecular weight cut-off of the support UF membrane was 20,000. The hydrolysis conditions were in agreement with our previous work [7]. Linear PEI with the molecular weight of 60,000 was purchased from ACROS.

The PEI solution was led tangentially over the hydrolyzed PAN support in a square channel using a peristaltic pump. The transmembrane pressure was kept at around $0.1 \mathrm{MPa}$ by means of a back pressure valve. As the PEI is positively charged, it was decided to place the cathode on the permeate side and the anode on the concentrate side of the membrane. An electric field may be applied when PEI solution was pumped into the capacitor cell and this may facilitate the adsorption of positive PEI on the negatively charged hydrolyzed PAN support. After adsorption for a period of $20 \mathrm{~min}$, the membrane was taken out, briefly rinsed with de-ionized water for approximately $5 \mathrm{~min}$ and dried in an oven at $50{ }^{\circ} \mathrm{C}$. The membranes obtained were characterized by the pervaporation of alcohol/water mixtures $(95: 5, \mathrm{~g} / \mathrm{g})$. The composition of the collected permeate was determined by gas chromatography (GC-14C, SHIMADZU). The separation factor was calculated from the quotient of the weight ratio of water and alcohol in permeate, $Y_{\mathrm{HOH}} / Y_{\mathrm{ROH}}$, and in feed, $X_{\mathrm{HOH}} / X_{\mathrm{ROH}}$ :

$\alpha=\frac{Y_{\mathrm{HOH}} / Y_{\mathrm{ROH}}}{X_{\mathrm{HOH}} / X_{\mathrm{ROH}}}$ 

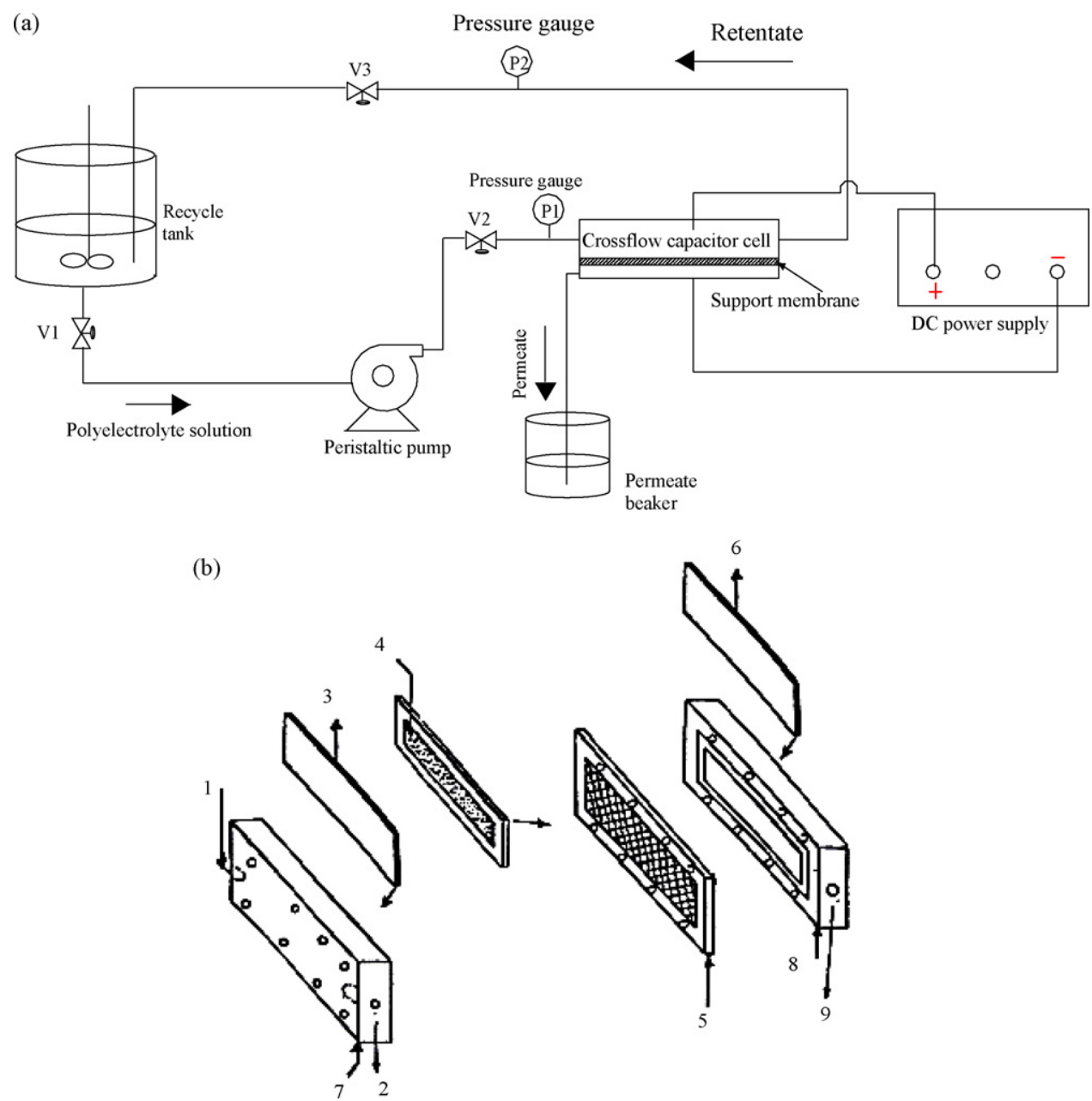

Fig. 2. Experimental apparatus for electric field-enhanced assembly of polyelectrolyte composite membrane. (a) Experimental setup; (b) crossflow capacitor cell: (1) polyelectrolyte solution inlet, (2) retentate outlet, (3) deployed stainless steel sheet, (4) support membrane, (5) porous support sheet, (6) deployed stainless steel sheet, (7) assembly A, (8) assembly B and (9) permeate outlet.

Atomic force microscopy (AFM) surface topography was performed in tapping mode by a Digital Nanoscope III AFM (Digital Instruments, Buffalo, NY).

\section{Results and discussion}

In order to clearly observe the effects of different assembly process on membrane pervaporation performance, only one PEI layer ( 0.5 bilayer) was deposited on the hydrolyzed
PAN support. Table 1 summarizes the characteristic properties of polyelectrolyte membranes assembled with different methods.

As shown in Table 1, after hydrolysis with $\mathrm{NaOH}$ solution, the hydrolyzed PAN support membrane showed a little pervaporation capacity. The water content in permeate and the flux were $9.8 \%$ and $17,850 \mathrm{~g} / \mathrm{m}^{2} \mathrm{~h}$, respectively. After statically adsorbing one PEI layer, the water content in permeate increased to $21.3 \%$, while the flux decreased to $13,780 \mathrm{~g} / \mathrm{m}^{2} \mathrm{~h}$. This suggested

Table 1

Comparison of polyelectrolyte composite membrane by depositing PEI with different methods

\begin{tabular}{|c|c|c|c|c|c|}
\hline Method & Assembly conditions & $\begin{array}{l}\text { Temperature } \\
\left({ }^{\circ} \mathrm{C}\right)\end{array}$ & $\begin{array}{l}\text { Water content in } \\
\text { permeate }(\%)\end{array}$ & $\begin{array}{l}\text { Separation } \\
\text { factor }\end{array}$ & $\begin{array}{l}\text { Permeate } \\
\text { flux }\left(\mathrm{g} / \mathrm{m}^{2} \mathrm{~h}\right)\end{array}$ \\
\hline Hydrolysis & Without PEI deposition & 60 & 9.80 & 2 & 17,850 \\
\hline Static PEI deposition & $\begin{array}{l}\text { Without pressure, static } \\
\text { adsorption } 20 \mathrm{~min}\end{array}$ & 60 & 21.30 & 5 & 13,780 \\
\hline Dynamic PEI deposition with dead-end system & Pressure: $0.1 \mathrm{MPa}, 20 \mathrm{~min}$ & 60 & 75.92 & 60 & 429 \\
\hline Dynamic PEI deposition with crossflow system & Pressure: $0.1 \mathrm{MPa}, 20 \mathrm{~min}$ & 60 & 48.63 & 18 & 10,240 \\
\hline Electric field-enhanced assembly with crossflow system & $0.1 \mathrm{MPa}, 30 \mathrm{~V}, 20 \mathrm{~min}$ & 60 & 87.91 & 138 & 357 \\
\hline Electric field-enhanced assembly with crossflow system & $0.1 \mathrm{MPa}, 30 \mathrm{~V}, 20 \mathrm{~min}$ & 75 & 94.11 & 304 & 514 \\
\hline
\end{tabular}

Hydrolysis conditions for PAN support membrane: $2 \mathrm{~N} \mathrm{NaOH}$, hydrolysis temperature, $65^{\circ} \mathrm{C}$; hydrolysis time, 60 min. Preparative conditions: only one PEI layer deposited, $0.25 \mathrm{wt} \%$ PEI ethanol solution $25^{\circ} \mathrm{C}$. Pervaporation conditions: downstream pressure $100 \mathrm{~Pa}$, EtOH content in feed solution $95 \mathrm{wt} \%$. 
pervaporation capabilities of the membranes were mainly due to the PEI layer deposited. It was also noted from Table 1 the selectivity of the membrane obtained from dynamic PEI deposition significantly improved with a dead-end cell. In this case, the water content in permeate and the flux could reach $75.92 \%$ and $429 \mathrm{~g} / \mathrm{m}^{2} \mathrm{~h}$, respectively. This indicated that the electrostatic adsorption of oppositely charged polyelectrolytes could be enhanced by the combination with an external pressure although the electrostatic force was the dominant formation reason. However, there is a clear difference between dead-end and crossflow system. In the case of dynamic crossflow filtration, the selectivity significantly declined because the unsteady polyelectrolytes absorbed on the membrane surface was frequently washed away due to the tangential flow. Consequently, the polyelectrolyte was much more difficult to attach on the support membranes. However, in the cases when electric field is applied during electrostatic adsorption, the pervaporation capacity greatly improved. With only one PEI deposited, the
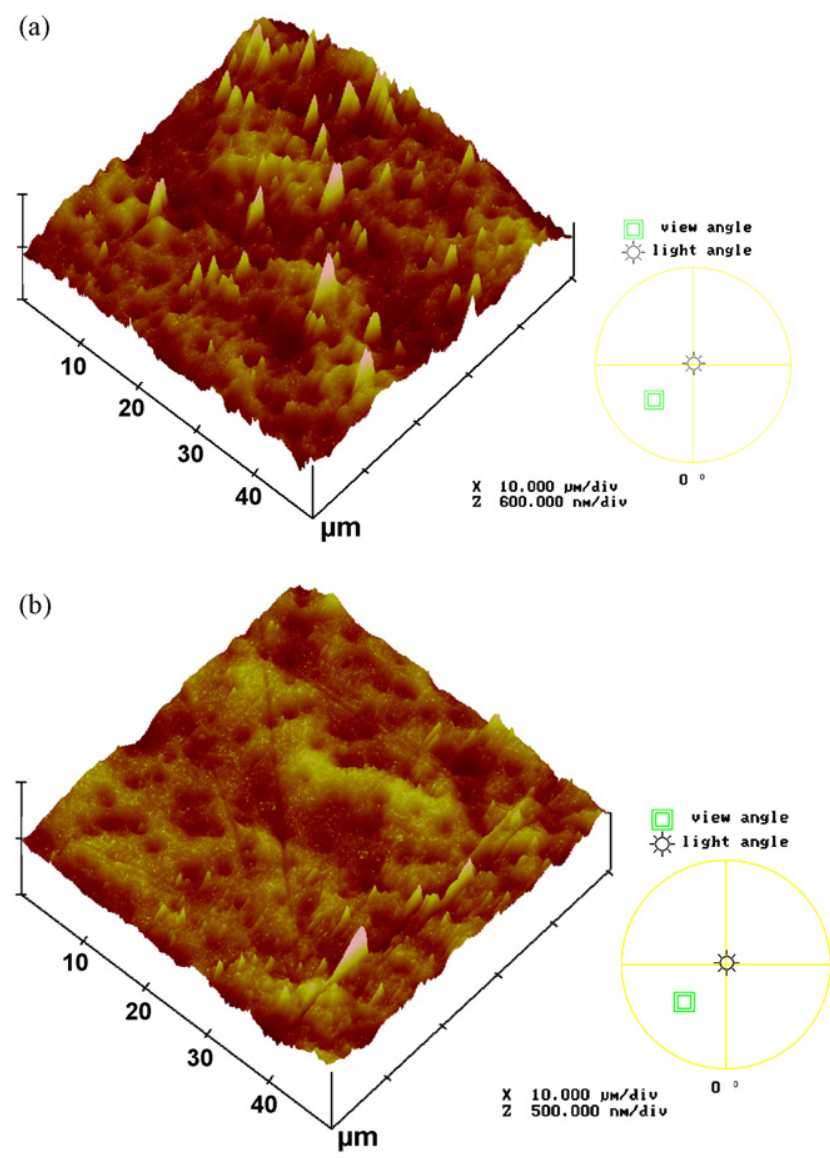

Fig. 3. AFM images of membrane surfaces. (a) Polyelectrolyte composite control membrane assembled by dynamic PEI deposition technique with dead-end system $(50 \mu \mathrm{m} \times 50 \mu \mathrm{m})$. (b) Polyelectrolyte composite membrane assembled by electric field-enhanced adsorption with crossflow system $(50 \mu \mathrm{m} \times 50 \mu \mathrm{m})$. Hydrolysis conditions for PAN support membrane: hydrolysis temperature, $65^{\circ} \mathrm{C}$; hydrolysis time, $60 \mathrm{~min}$. Preparative conditions for the membrane assembled by dynamic assembly: 0.1 MPa dynamic pressure, 20 min filtration time, $0.25 \mathrm{wt} \% \mathrm{PEI}-\mathrm{ethanol}$ solution, $25^{\circ} \mathrm{C}$. Preparative conditions for the membrane assembled by electric filed-enhanced assembly: $0.1 \mathrm{MPa}$ dynamic pressure, 30 voltage DC electric field, $20 \mathrm{~min}$ action time, $0.25 \mathrm{wt} \%$ PEI-ethanol solution, $25^{\circ} \mathrm{C}$. membranes obtained had a separation factor of 304 and a permeate flux of $514 \mathrm{~g} / \mathrm{m}^{2} \mathrm{~h}\left(75^{\circ} \mathrm{C}\right)$ for pervaporation of $95 \mathrm{wt} \%$ ethanol-water mixture. It was also noted that both permeate flux and water content in permeate increased with the increase in feed temperature. In comparison with those previous studies of 60 bilayers [1-3], the substantial reduction of the number of polyion depositions would possibly make the manufacturing of polyelectrolyte composite membranes practically viable. In fact, under the action of the electric field, the charged polyelectrolytes rapidly and orientedly migrated toward the support and in turn resulted in a rapid formation of uniform and dense membrane. Furthermore, the orientation of charged polyelectrolytes could be arranged in a regular order. Therefore, the separation factor of the membranes obtained from electric fieldenhanced assembly largely increased even compared with the membranes assembled by dynamic assembly with dead-end system. Fig. 3 shows the AFM images of the two membranes with better performances. The two membranes were assembled under the crossflow electric field system and dynamic pressure deadend system, respectively. Compared Fig. 3(a) with (b), it was clearly demonstrated that the regularity of membrane surfaces obtained from the new method was much higher than that of the membranes obtained dynamic assembly with dead-end system. The values of mean roughness $(\mathrm{Ra})$ were also obtained based on $10.0 \mu \mathrm{m} \times 10.0 \mu \mathrm{m}$ scan area. The Ra of the membranes decrease from 17.281 to $7.277 \mathrm{~nm}$ after using electric field-enhanced assembly. This further confirmed that the structure order became much more regular with the action of electric field.

\section{Conclusions}

In this study, a new method of electric field-enhanced assembly was proposed to prepare polyelectrolyte composite membranes. It was found that the combination of electrostatic adsorption of oppositely charged polyelectrolytes with the external electric force could facilitate the assembly of polyelectrolyte composite membranes. It was demonstrated that using this technique a relatively good permselectivity could be achieved with only one PEI layer deposited on a hydrolyzed PAN support, which was much less than the number of cycles used in the literature (e.g. 60). In the case of pervaporation of $95 \mathrm{wt} \%$ ethanol/water mixture, the membranes obtained had a separation factor of 304 and a permeate flux of $512 \mathrm{~g} / \mathrm{m}^{2} \mathrm{~h}\left(75^{\circ} \mathrm{C}\right)$. Finally, the observation of AFM images further confirmed that the structure order became much more regular with the action of electric field. This might lead to much better membrane performance.

\section{Acknowledgements}

This work was supported by the Beijing NOVA Programme (No. 2006B13), the Key Project of the Natural Science Foundation of Beijing (No. 07h0003), the National Basic Research Program of China (No. 2003CB615701) and the Special Funding Project of the Beijing Municipal Commission of Education, PR China (No. 05005999200601). 


\section{References}

[1] L. Krasemann, A. Toutianoush, B. Tieke, Self-assembled polyelectrolyte multilayer membranes with highly improved pervaporation separation of ethanol/water mixtures, J. Membr. Sci. 181 (2001) 221-228.

[2] A. Toutianoush, B. Tieke, Pervaporation separation of alcohol/water mixtures using self-assembled polyelectrolyte multilayer membranes of high charge density, Mater. Sci. Eng. C 22 (2002) 459-463.

[3] A. Toutianoush, L. Krasemann, B. Tieke, Polyelectrolyte multilayer membranes for pervaporation separation of alcohol/water mixtures, Colloids Surf. A 198-200 (2002) 881-889.

[4] D.M. Sullivan, M.L. Bruening, Ultrathin cross-linked polyimide pervaporation membranes prepared from polyelectrolyte multilayers, J. Membr. Sci. 248 (2005) 161-170.
[5] Z. Zhu, X. Feng, A. Penlidis, Self-assembled nano-structured polyelectrolyte composite membranes for pervaporation, Mater. Sci. Eng. C 26 (2006) $1-8$.

[6] G. Zhang, W. Gu, S. Ji, Z. Liu, Y. Peng, Z. Wang, Preparation of polyelectrolyte multilayer membranes by dynamic layer-by-layer process for pervaporation separation of alcohol/water mixtures, J. Membr. Sci. 280 (2006) 727-733.

[7] G. Zhang, H. Yan, S. Ji, Z. Liu, Self-assembly of polyelectrolyte multilayer pervaporation membranes by a dynamic layer-by-layer technique on a hydrolyzed polyacrylonitrile ultrafiltration membrane, J. Membr. Sci. 292 (2007) 1-8. 